Journal of

Cancer Research and Therapeutic Oncology

\title{
Apatinib Monotherapy as a Second-Line Treatment for Patients with Advanced Biliary Tract Cancer: a Retrospective Single-Center Observational Study
}

\author{
Yue Zhao ${ }^{1, *, *}$, Wenqian $\mathrm{Gu}^{2, *}$, Jianmei Li ${ }^{1}$, Chunyuan Tian ${ }^{1}$
}

${ }^{1}$ Department of Oncology, Qinhuangdao Fourth Hospital, 64 Guangming Rd, 066000, Hebei Province, China

${ }^{2}$ Department of Clinical Medicine, Aarhus University, Palle Juul-Jensens Boulevard 165, 8200 Aarhus N, Denmark

${ }^{\star}$ Corresponding author: Yue Zhao, Department of Oncology, Qinhuangdao Fourth Hospital, 64 Guangming Rd, 066000, Hebei Province, China, Tel.: +86 18033505576, E-mail: chinaxh2007@163.com

\# These authors contributed equally to this work

Received Date: December 03, 2019 Accepted Date: December 27, 2019 Published Date: December 30, 2019

Citation: Yue Zhao (2019) Apatinib Monotherapy as a Second-Line Treatment for Patients with Advanced Biliary Tract Cancer: a Retrospective Single-Center Observational Study. J Cancer Res Therap Oncol 7: 1-11.

\begin{abstract}
Biliary tract cancer is an aggressive gastrointestinal malignancy with a dismal prognosis. Combined chemotherapy with gemcitabine and cisplatin is the gold standard of first-line treatment for patients with advanced biliary tract cancer, while second-line therapy remains inconclusive. We aimed to evaluate the effects of apatinib as a second-line regimen in patients with advanced biliary tract cancer. We retrospectively analyzed 18 patients with advanced biliary tract cancer who failed first-line therapy with gemcitabine alone or gemcitabine combined with cisplatin and received second-line treatment with apatinib from June 2016 to October 2017. Adverse events, progression-free survival, objective response rate, and disease control rate were documented and evaluated. All patients were followed up until progression of the disease. All patients were included in the efficacy analysis, including 6 drop-out cases. The 1-month tumor response assessment showed an objective response rate of $33 \%$ and a disease control rate of $72 \%$. At a 6 -month follow-up, a disease control rate of $44 \%$ was observed. The cohort achieved a median progression-free survival of 8 months; for gallbladder carcinoma of 2.5 months; for cholangiocarcinoma of 8 months. Fatigue, hypertension, and proteinuria were the most frequently occurring apatinib-related toxicities. Nine cases of grade 3 adverse effects were recorded, and no bleeding cases or treatment-related death were observed. This retrospective analysis showed favorable outcome and manageable toxicities of apatinib treatment in a second-line setting in patients with advanced biliary tract cancer. Perspective studies are needed to confirm the results from this study.
\end{abstract}

Keywords: Apatinib mesylate; second-line treatment; biliary-tract cancer; targeted therapy; anti-angiogenesis

Abbreviations: BTC: Biliary tract cancer

What's New: This is the first report to demonstrate that Apatinib may have a beneficial effect in patients with advanced biliary tract cancer in a second-line setting. The result shows a progressive-free survival of 8 months, which provides a rational for further perspective study.

(c)2019 The Authors. Published by the JScholar under the terms of the Creative Commons Attribution License http://creativecommons.org/licenses/ by $/ 3.0 /$, which permits unrestricted use, provided the original author and source are credited. 


\section{Introduction}

Biliary tract cancer (BTC) is a lethal malignancy encompassing gallbladder carcinoma and cholangiocarcinoma. The latter is further subdivided into intrahepatic cholangiocarcinoma and extrahepatic cholangiocarcinoma [1]. Though it is less common in the Western world, where the annual incidence is 0.35 to 2 per 100,000 , the prevalence in Hispanic and Asian populations is much higher [2]. For instance, the incidence may be up 40-fold higher in China than in the United Kingdom $[3,4]$.

At present, a satisfactory outcome of BTC hinges on early diagnosis and complete surgical resection. However, due to its slow-growing nature and non-specific symptomatology, BTC is often diagnosed at an advanced stage when surgical options are limited $[5,6]$. Even in patients undergoing successful resection, recurrence rates may reach $49-64 \%$, and most cases will eventually progress to metastatic disease, which highlighting the importance of advancing adjuvant therapies [7-9].

According to the guideline of the National Comprehensive Cancer Network, Version 2.2019, standard first-line chemotherapeutic regimens for BTC include gemcitabine-based or gemcitabine-cisplatin combination therapy for the metastatic or unresectable disease [10]. However, no clear guidance exists for second-line treatment following disease progression under first-line chemotherapy. Many clinical trials are currently being conducted to evaluate the efficacy of a multitude of agents targeting the molecular level in second-line settings [11]. However, the results of these trials have not yet been published. Angiogenesis, a physiological process through which new blood vessels form from pre- existing vessels is responsible for tumor growth and disease progression $[12,13]$. In recent years, anti-angiogenic treatment has become an essential tool in the armamentarium against advanced cancers. Vascular endothelial growth factor (VEGF) and its cognate receptor, VEGFR-2 is recognized as the most prominent regulators of angiogenesis [14], and have therefore become the main target of current antiangiogenic agents. Thus, strategies focus on antiangiogenesis therapy via VEGF pathway inhibition might have clinical benefits in the treatment of patients with advanced BTC [15]. A number of agents targeting the VEGF/VEGFR signaling pathway, including bevacizumab, aflibercept, and ramucirumab, have been developed and approved for several indications by the U.S. Food and Drug Administration [13]. VEGF is over expressed in $40-75 \%$ of patients with BTC [16]. In a phase II trial, bevacizumab was added to second-line chemotherapy for advanced BTC and reported to have a modest benefit as well as a tolerable safety profile [17]. Moreover, in another phase II trial of treatment of advanced BTC, ramu- cirumab showed limited activity but still infrequent grade 3-4 treatment-related adverse events (AEs) when combined with pembrolizumab in a second-line setting [18].

Apatinib is an oral tyrosine kinase inhibitor selectively inhibiting VEGFR-2, thereby suppressing tumor growth by obstructing angiogenesis. It has been approved in China for advanced or metastatic gastric cancer and has shown promising therapeutic effects against a variety of other cancer types, including ovarian cancer [19], breast cancer [20-22], lung cancer [23, 24], hepatocellular carcinoma [25-27], sarcoma [28-30], and thyroid cancer [31,32]. Nevertheless, research evaluating the effect of apatinib on advanced BTC is limited. As a broad-spectrum anticancer agent, apatinib has been a frequent option for patients with advanced BTC after first-line treatment failure in our hospital at the discretion of the treating physicians and showed favorable effects.

In the present study, we hypothesized that apatinib is an effective second-line regimen for the treatment of patients with advanced BTC. Therefore, we retrospectively assessed the effects of apatinib monotherapy in a second-line setting after the failure of first-line systemic chemotherapy with gemcitabine or gemcitabine-cisplatin in patients with advanced BTC. 98

\section{Patients and Methods}

\section{Eligibility criteria}

The study was conducted retrospectively on patients treated between June 2016 and October 2017 at Qinhuangdao Fourth Hospital, China. Eligibility criteria included 1) age $>18$ years old, 2) pathologically confirmed advanced BTC with contrast-enhanced CT or magnetic resonance imaging (MRI) scan, 3) disease progressed after first-line chemotherapy - gemcitabine or gemcitabine-cisplatin, 4) started apatinib monotherapy as second-line regimen after first-line failure, 5) Karnofsky Performance Scale (KPS) >80. Key exclusion criteria were: pregnant or lactating women; History of other malignancies except cured basal cell carcinoma of the skin and carcinoma in-situ of uterine cervix; serious respiratory, cardiovascular or kidney disease; prior VEGFR inhibitor treatment.

\section{Treatment Methods}

Following disease progression after first-line treatment, patients were offered apatinib at a dose of $500 \mathrm{mg}$ once daily until disease progression or intolerable. One dose reduction (to $250 \mathrm{mg}$ ) due to drug-related toxicity was allowed. Dose-limiting toxicity was defined as possibly or definitely drug-related grades 
3-4 toxic responses. Apatinib was provided by Jiangsu Hengrui Medicine Co., Ltd and was administered orally. One treatment cycle was 28 days long.

\section{Evaluation of efficacy and safety}

Clinical and radiologic evaluations were conducted at baseline and at 1 month; thereafter every 2 months or whenever clinically indicated until disease progression. At 1 month and 6 months following apatinib administration, tumor response was assessed as complete response (CR), partial response (PR), stable disease (SD), and progressive disease (PD) according to the Response Evaluation Criteria in Solid Tumors (RECIST) 1.1. Definitions of the four categories of tumor response are listed in Table 1. Progression-free survival (PFS) was defined as the length of time from enrollment (1st apatinib administration) to investigator-assessed disease progression or death from any cause, whichever occurred first. Objective response rate (ORR) was defined as the percentage of patients with an objective response among all cases and calculated as the CR rate plus the PR rate. Disease control rate (DCR) was defined as the percentage of patients with CR or PR, or SD. Treatment efficacy was evaluated by CT or MRI. AEs and severe adverse events (SAE) were classified and graded according to the National Cancer Institute's Common Terminology Criteria for Adverse Events (CTCAE), v4.03. Apatinib-related toxicities were evaluated according to the National Cancer Institute Common Toxicity Criteria version 4.0. A schematic illustration of the study overview is shown in Figure 1 .

\begin{tabular}{|l|l|}
\hline Category & Definition \\
\hline Complete response (CR) & Complete disappearance of all target lesions \\
\hline Partial response (PR) & $\geq 30 \%$ decrease in tumor diameter from baseline \\
\hline Stable disease (SD) & Small changes that do not meet the above criteria \\
\hline Progressive disease (PD) & $>20 \%$ increase in tumor diameter from baseline \\
\hline
\end{tabular}

Table 1: Four response categories

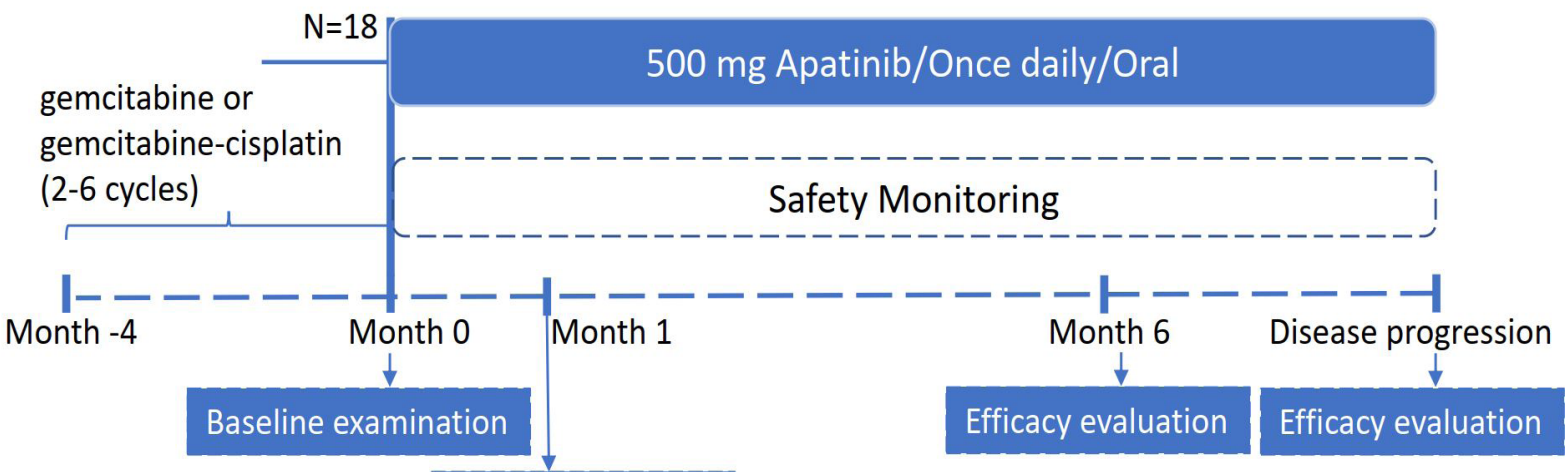

Efficacy evaluation

Enrolment Period: June 2016 - Oct 2017

Figure 1: Study overview 


\section{Statistical Analysis}

Data analysis was performed with Graph Pad Prism Software Version 7.04 (Graph Pad Software, San Diego, CA, USA). Kaplan-Meier survival curves were used for PFS estimation. P-values $<0.05$ were considered statistically significant.

\section{Results}

\section{Patient characteristic}

Before data cutoff, 18 patients with advanced BTC received their first dose of apatinib, including 12 patients (67\%) with cholangiocarcinoma and 6 patients (33\%) with gallbladder carcinoma. The metastatic site included liver, retroperitoneal node, lungs, and supraclavicular lymph node. All pathological results were confirmed at Qinhuangdao Forth Hospital, China. Patient characteristics at baseline are summarized in Table 2. Within 4 months before apatinib administration, all patients had received gemcitabine as a single agent or in combination with cisplatin for 2-6 cycles (only 3 patients who were over 60 years old received gemcitabine monotherapy; all other patients received the combined regimen) and, unfortunately, the disease progressed. The patients' median age was 55 years and males counted for $78 \%$. Eight cases (44\%) were post-surgery recurrence, while the other $10(56 \%)$ were initially diagnosed, patients. All patients started apatinib at a dosage of $500 \mathrm{mg}$; in 6 patients (33\%), the apatinib dose was reduced to $250 \mathrm{mg}$ daily within the first10 days due to intolerable toxicity.

\section{Evaluation of efficacy}

After 1 month of treatment with apatinib, all patients who had received at least one dose of apatinib were analyzed for tumor response, including 2 withdrawal cases. Although no patient was rated as having CR, PR occurred in 6 patients, including in 2 with gallbladder carcinoma and 4 with cholangiocarcinoma. Seven patients experienced SD of whom 2 had gallbladder carcinoma and the other 5 cholangiocarcinomas. In addition, 3 patients suffered from PD, including 2 with gallbladder carcinoma and 1 with cholangiocarcinoma. The ORR was 33\%, while the DCR was $72 \%$. At 6-month follow-up, except for another 4 discontinuations, there were 8 patients remained SD ( 2 with gallbladder carcinoma, 6 with cholangiocarcinoma) and 4 patients ( 2 with gallbladder carcinoma, 2 with cholangiocarcinoma) had
PD, which resulted in a DCR of $44 \%$. All patients were followed up until progression of the disease. All 18 patients, including 6discontinued cases, were counted for Kaplan-Meier analysis for PFS. The median PFS was 8 months for the whole population; it was 2.5 months for gallbladder carcinoma and 8 months for cholangiocarcinoma. Kaplan-Meier curve for PFS presenting the whole cohort is depicted in Figure 2A; Figure 2B compares the curves for gallbladder carcinoma and cholangiocarcinoma.

Image data for a 65-year-old male patient are shown in Figure 3A, 3B and 3C. He was diagnosed with cholangiocarcinoma and underwent complete resection in August 2013. Unfortunately, pulmonary metastasis occurred in Aril 2016 and he had received gemcitabine-cisplatin for 2 cycles before PD was diagnosed. He started to take $500 \mathrm{mg}$ of apatinib orally per day from 12 June 2016. One month later, the size of the targeted lesion had decreased from approximately $6.5^{\star} 4 \mathrm{~cm}$ to $4.6^{\star} 3.5 \mathrm{~cm}$. Thereafter, the tumor was further shrunk to approximately $3.2^{\star} 2$ $\mathrm{cm}$ at 2.5 months after treatment. Dose reduction was needed due to fatigue and hypertension. Following symptomatic treatment, symptoms were palliated. The disease progressed in Feb 2017, indicating a PFS of 8 months.

Evaluation of safety and toxicity Fatigue, hypertension, and proteinuria were the most frequently encountered toxicities in this study. Up to 13 of the patients (72\%) suffered from fatigue, 11 patients (61\%) developed hypertension, while 8 patients (44\%) had proteinuria. Most AE cases were grade 1 or 2, see Table 3. Four patients required apatinib discontinuation due to grade 3 AEs, including 2 hypertension cases, 1 diarrhea and 1 hand-foot syndrome case; and all recovered after drug withdrawal. Two patients gave up cancer treatment due to grade 3 hypertension and fatigue and for personal reasons. No bleeding cases, grade 4 AEs, or drug-related deaths occurred.

For hypertension, patients were given valsartan at a dose of $80 \mathrm{mg}$ twice daily or $80 \mathrm{mg}$ of valsartan in combination with $5 \mathrm{mg}$ of levamlodipine once daily. Most diarrhea cases did not involve abdominal pain, and loperamide was prescribed. For grade $1 \& 2$ hand-foot syndrome, lanolin cream was applied locally to alleviate pain, ulcer, and desquamation. Loperamide had a better effect on diarrhea than montmorillonite and Bifidobacterium. 


\begin{tabular}{|c|c|c|}
\hline \multicolumn{2}{|l|}{ Characteristics } & \multirow{2}{*}{$\begin{array}{l}\text { Number of patients } \\
14\end{array}$} \\
\hline \multirow{2}{*}{ Sex } & Male & \\
\hline & Female & 4 \\
\hline \multirow{4}{*}{ Age (years) } & Median & 55 \\
\hline & $40-50$ & 4 \\
\hline & $50-60$ & 9 \\
\hline & $60-70$ & 5 \\
\hline \multicolumn{3}{|l|}{ Metastatic site } \\
\hline \multirow{4}{*}{$\begin{array}{l}\text { From cholangiocar- } \\
\text { cino ma }\end{array}$} & Liver & 2 \\
\hline & Retroperitoneal node & 5 \\
\hline & Lungs & 3 \\
\hline & Supraclavicular lymph node & 2 \\
\hline \multirow{2}{*}{$\begin{array}{ll}\text { From } & \text { gall- } \\
\text { bladder } & \text { can- } \\
\text { cer } & \\
\end{array}$} & Liver & 2 \\
\hline & $\begin{array}{l}\text { Gallbladder (locally ad- } \\
\text { vanced) }\end{array}$ & 4 \\
\hline \multicolumn{3}{|c|}{ Initial treatment/ recurrence } \\
\hline \multicolumn{2}{|c|}{ Initial treatment } & 10 \\
\hline \multicolumn{2}{|c|}{ Post-surgery Recurrence } & 8 \\
\hline \multicolumn{3}{|c|}{ Gemcitabine (first-line medicine) therapy } \\
\hline \multicolumn{2}{|c|}{ Monotherapy } & 3 \\
\hline \multicolumn{2}{|l|}{ Gemcitabine-cisplatin } & 15 \\
\hline \multicolumn{3}{|c|}{ Initial dosage of apatinib (mg) } \\
\hline \multicolumn{2}{|c|}{500} & 12 \\
\hline \multicolumn{2}{|c|}{250 (dosage reduced from 500 within the first 10 days) } & 6 \\
\hline
\end{tabular}

Table 2: Patient characteristics

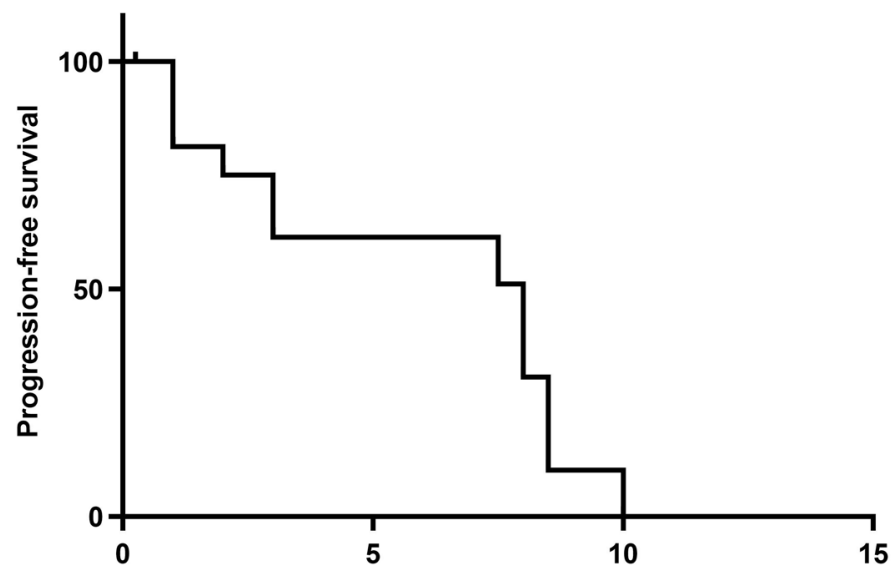

Figure 2A: Overall progression-free survival (PFS) for patients with advanced biliary tract cancer treated with apatinib monotherapy as a second-line regimen. The median PFS was 8 months. 


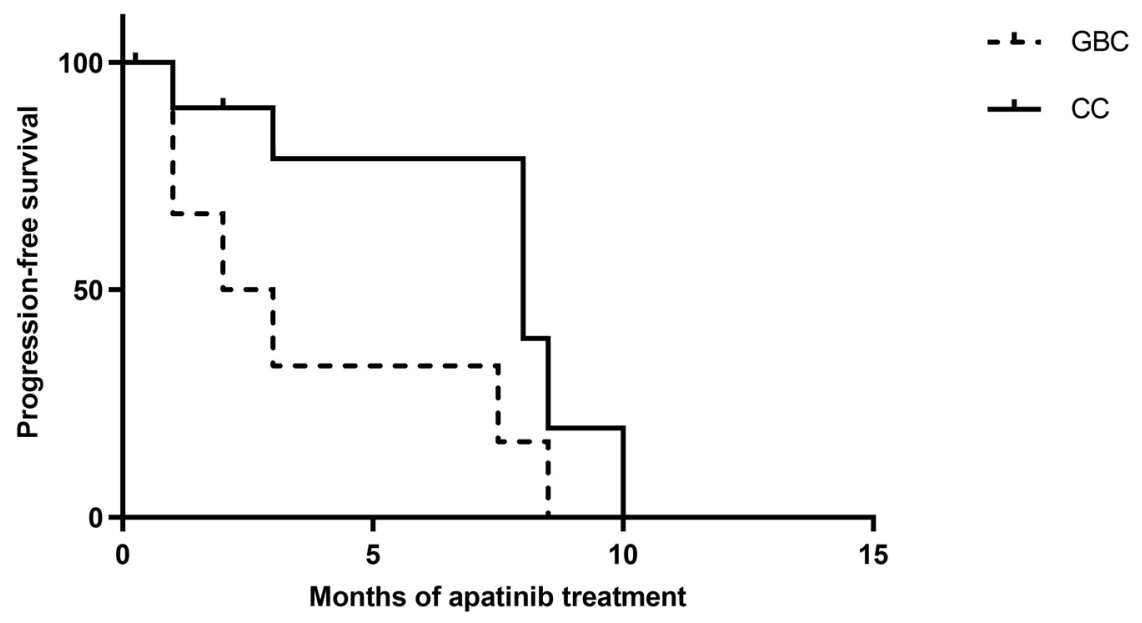

Figure 2B: Progression-free survival (PFS) for patients with advanced cholangiocarcinoma (CC) and gallbladder carcinoma (GBC) treated with apatinib monotherapy as a second-line regimen. The median PFS in GBC group was 2.5 months and in CC group was 8 months, $\mathrm{p}=0.052$
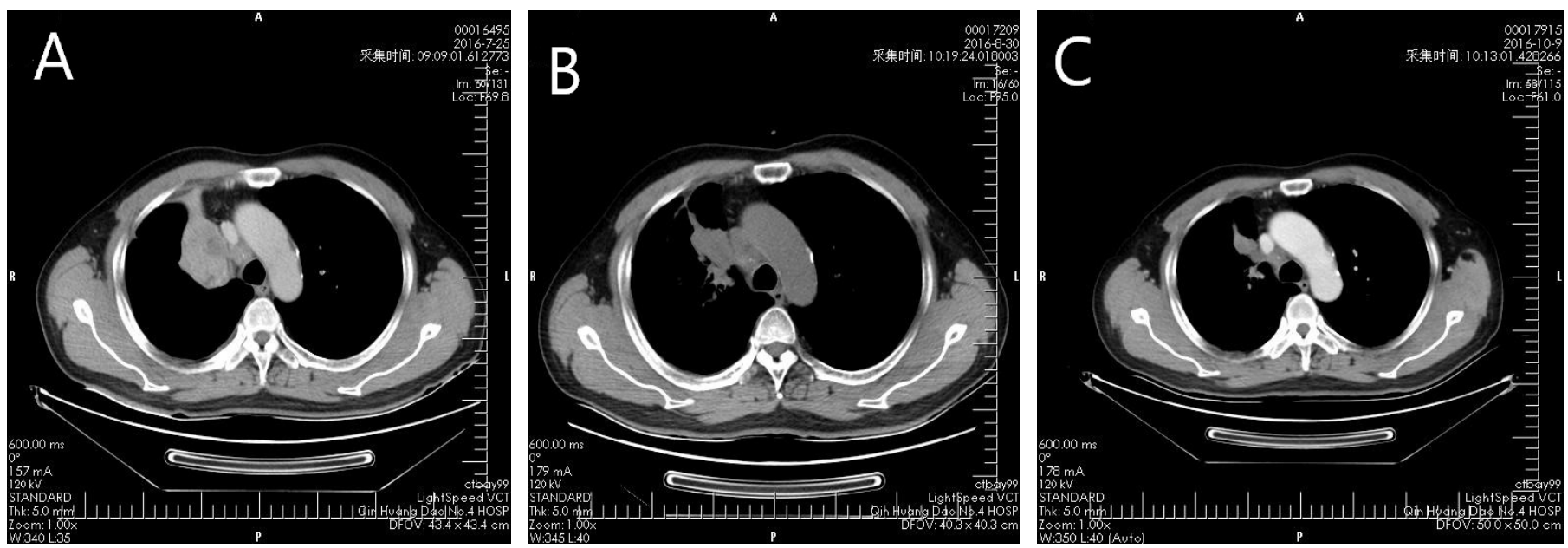

Figure 3: Images of a 65-year-old male patient with cholangiocarcinoma after treatment with apatinib monotherapy. Contrastenhanced CT at baseline (A) showed an approximately $6.5^{\star} 4 \mathrm{~cm}$ cholangiocarcinoma. CT images at 1 month (B) and 2.5 months (C) after baseline showed the tumor shrunk to $4.6^{\star} 3.5 \mathrm{~cm}$ and $3.2^{\star} 2 \mathrm{~cm}$, respectively.

\begin{tabular}{|l|l|l|l|}
\hline \multirow{2}{*}{ Adverse events } & Total, n\% & Grade & G3 \\
\cline { 3 - 5 } & & G1-G2 & 4 \\
\hline Hypertension & $11(61 \%)$ & 7 & 1 \\
\hline Diarrhea & $6(33 \%)$ & 5 & 1 \\
\hline $\begin{array}{l}\text { Hand - fo ot } \\
\text { syndrome }\end{array}$ & $5(28 \%)$ & 4 & 1 \\
\hline Proteinuria & $8(44 \%)$ & 7 & 2 \\
\hline Fatigue & $13(72 \%)$ & 11 & - \\
\hline Liver dysfunction & $2(11 \%)$ & 2 & - \\
\hline Anemia & $2(11 \%)$ & 3 & - \\
\hline Neutropenia & $2(11 \%)$ & 2 & - \\
\hline Thrombocytopenia & $1(6 \%)$ & 1 & \\
\hline
\end{tabular}

Table 3: Treatment-related adverse events. 


\section{Discussion}

To our knowledge, this is the first study evaluating the efficacy and safety profile of apatinib as a second-line treatment in patients with advanced BTC. The median PFS was 8 months, while the 1 -month ORR was $33 \%$ and DCR was $72 \%$, the 6-month DCR was $44 \%$. Furthermore, apatinib had a manageable toxicity profile.

Even though surgery is potentially curative, BTC has a high recurrence rate due to its aggressive nature. The vast majority of patients therefore also received systemic chemotherapy, where the current standard therapy is gemcitabine plus cisplatin [33]. The strategy for second-line treatment after failure of firstline chemotherapy in advanced BTC remains unclear as research in this field is insufficient. Twenty-five studies were evaluated in a systematic review, including 14 phases II clinical trials, 9 retrospective studies, and 2 case reports to determine the level of evidence for the use of second-line chemotherapy in patients with BTC; a mean PFS of 3.2 was observed [34]. Furthermore, a multicenter survey of 174 patients reported a median PFS of 3.0 months and a pooled analysis of 499 patients reported a median PFS of 3.1 months after second-line chemotherapy [35]. These results indicate a limited value of chemotherapy as a second-line treatment for advanced BTC. Efforts devoted to establishing second-line therapies are therefore pivotal to determine what constitutes successful management of advanced BTC.

Apatinib is a highly selective VEGFR-2 inhibitor. An in vitro study demonstrated that VEGF/VEGFR-2 was significantly over-expressed in the intrahepatic tissue of patients with cholangiocarcinoma and apatinib suppressed the anti-apoptotic process induced by VEGF signaling and promoted cell death in intrahepatic cholangiocarcinoma cell lines (RBE and SSP25) [36]. Meanwhile, in cholangiocarcinoma cell lines (QBC939 and TFK-1), apatinib was reported to inhibit VEGF-mediated cell migration and invasion by inhibiting VEGF- 2/RAF/MEK/ERK and P13K/AKT pathways [37]. Furthermore, in a case report, apatinib was used for a 23-year-old female with advanced unresectable intrahepatic cholangiocarcinoma as second-line treatment and a PFS of 6 months were observed [38].

To further study the response to apatinib, we retrospectively analyzed the safety and efficacy data of apatinib as a second-line regimen in patients with advanced BTC, revealing a median PFS of 8 months. In the present study, the outcome of apatinib monotherapy was associated with a longer median PFS time than what has previously been reported with chemotherapy [34, 35, 39], and our findings mirror the above-mentioned result from the case report [38]. This indicates that targeted therapy, especially with agents targeting VEGF pathway, might constitute a breakthrough in establishing a second-line treatment for advanced BTC.

Though both cholangiocarcinoma and gallbladder carcinoma fall under BTC, they may be in fact distinct diseases with differences in prognosis and patterns of recurrence [40].

Additionally, immunohistochemical analysis for BTC samples has revealed the tumor somatic variants and genomic heterogeneity between the two diseases [41]. In this study, cholangiocarcinoma seemed more responsive to apatinib monotherapy than gallbladder carcinoma, as the median PFS was 8 months in patients with cholangiocarcinoma compared with 2.5 months in patients with gallbladder carcinoma. The arbitrary significance level was not fully reached $(\mathrm{P}=0.0502)$. Still, cholangiocarcinoma showed a clear tendency towards a better survival outcome than gallbladder carcinoma, which deserves more indepth studies to determine the underlying mechanism.

Previous studies with VEGF inhibitors across a variety of tumor types established a set of AEs attributed to antiangiogenic therapy, the most well-documented of which were hypertension, arterial thromboembolic events, proteinuria, bowel perforation, reversible posterior leukoencephalopathy syndrome wound complications, and hemorrhage $[13,42]$. In the present study, the majority of treatment-related AEs were fatigue, hypertension, proteinuria, diarrhea, and hand-foot syndrome, which is consistent with prior findings. We found somewhat higher rates of grade 3 AEs ( 9 cases) than previous studies and it's with other tumor types as there are few studies on BTC, which might due to the compromised general status of advanced BTC patients. However, most of these AEs could be relieved by dose interruptions and reductions, followed by careful symptomatic treatment. No bleedingcases or treatment-related death occurred.

A multitude of trials reported using lapatinib at a dose level of $750 \mathrm{mg}$ or $850 \mathrm{mg}$. However, we found that BTC patient was intolerant to such high dosages in practice. In the present study, a dose of $500 \mathrm{mg}$ was used based on the patient's age, body weight, and general status; in 6 patients, the dose was reduced to $250 \mathrm{mg}$ daily due to intolerability. Several limitations of the present study should be acknowledged. First, the sample size was relatively small and the drop-out rate was high. Six out of 18 patients withdrew from the study due to AEs, which could bias the efficacy results. Second, no overall survival (OS) data were obtained. Even though PFS was widely accepted as an adequate surrogate and even more meaningful than OS [43, 44], OS 
remains important for efficacy prediction. Another limitation is the absence of a control group to control for confounding from external factors. Therefore, large, well-designed, randomized controlled trials are needed to confirm these results.

In conclusion, the results of the present study reinforce the hypothesis that apatinib may have the potential to act as an effective second-line agent in the treatment of advanced BTC with a manageable safety profile. However, further randomized controlled studies are needed to verify the results obtained from this single-center, retrospective study.

\section{Author Contribution}

Conception and design: Yue Zhao

Collection and assembly of data: Yue Zhao, Jianmei Li, and Chunyuan Tian

Data analysis and interpretation: Wenqian $\mathrm{Gu}$ and Yue Zhao

Manuscript writing: Wenqian $\mathrm{Gu}$, Yue Zhao, Jianmei $\mathrm{Li}$, and Chunyuan Tian

Final approval of manuscript: Yue Zhao, Wenqian Gu, Jianmei $\mathrm{Li}$, and Chunyuan Tian

\section{Ethical Statement}

Approval for this study was obtained from the ethics committee of Qinhuangdao Forth Hospital, China; all procedures performed in this study were in accordance with the ethical standards of the institutional and national research committees and with the principles of the Declaration of Helsinki.

\section{Disclosure}

The authors declare that they have no conflicts of interest in this work.

\section{Acknowledgments}

The authors would like to thank Associate Professor, Chunsen $\mathrm{Wu}$, from Department of Clinical Research, the University of Southern Denmark and Odense University Hospital, for his untiring help and valuable assistance in statistical analysis.

\section{Data Availability statement}

The data that support the findings of this study are available from the corresponding author upon reasonable request.

C
$\mathrm{m}$




\section{Reference}

1. Ghidini M, C Pizzo, A Botticelli, JC Hahne, R Passalacqua, G Tomasello, et al. (2019) Biliary tract cancer: current challenges and future prospects. Cancer 291 Manag Res 11: 379-388.

2. Razumilava N and GJ Gores (2014) Cholangiocarcinoma. Lancet 383: 2168-2179.

3. Bridgewater JA, KA Goodman, A Kalyan, and MF Mulcahy, Biliary Tract (2016) Cancer: Epidemiology, Radiotherapy, and Molecular Profiling. Am Soc Clin Oncol 296 Educ Book 35: 194203.

4. Patel $T$ (2001) Increasing incidence and mortality of primary intrahepatic cholangiocarcinoma in the United States. Hepatology 33: 1353-1357.

5. Rakic M, L Patrlj, M Kopljar, R Klicek, M Kolovrat, et al. (2014) Gallbladder cancer. Hepatobiliary Surg Nutr 3: 221-226.

6. Guglielmi A, A Ruzzenente, T Campagnaro, S Pachera, A Valdegamberi, et al. (2009) Intrahepatic cholangiocarcinoma: prognostic factors after surgical resection. World J Surg 33: 1247 1254.

7. Blechacz B (2017) Cholangiocarcinoma: Current Knowledge and New Developments. Gut Liver 11: 13-26.

8. Wang Y, J Li, Y Xia, R Gong, K Wang, et al. (2013) Prognostic nomogram for intrahepatic cholangiocarcinoma after partial hepatectomy. J Clin Oncol 31: 1188-1195.

9. Hundal R and EA Shaffer (2014) Gallbladder cancer: epidemiology and outcome. Clin 312 Epidemiol 6: 99-109.

10. Benson AB, MI D'Angelica, DE Abbott, TA Abrams, SR Alberts, et al. (2019) Guidelines Insights: Hepatobiliary Cancers, Version 2.2019. J Natl Compr Canc Netw 17: 302-310.

11. Morizane C, M Ueno, M Ikeda, T Okusaka, H Ishii, and J Furuse (2018) New developments in systemic therapy for advanced biliary tract cancer. Jpn J Clin 322 Oncol 48: 703-711.

12. Folkman J (2007) Angiogenesis: an organizing principle for drug discovery? Nat Rev 324 Drug Discov 6: 273-286.

13. Zhao Y and AA Adjei (2015) Targeting Angiogenesis in
Cancer Therapy: Moving Beyond Vascular Endothelial Growth Factor. Oncologist 20: 660-673.

14. Lee, SH, D Jeong, YS Han, and MJ Baek (2015) Pivotal role of vascular endothelial growth factor pathway in tumor angiogenesis. Ann Surg Treat Res 89: 1-8.

15. Simone V, O Brunetti, L Lupo, M Testini, E Maiorano, M Simone, et al. (2017) Targeting Angiogenesis in Biliary Tract Cancers: An Open Option. Int J Mol Sci. 18.

16. Zheng W, J Ying, Y Zhou, Z Lu, K Min, W Wang, et al. (2019) The Efficacy and Safety of First-line Chemotherapies for Advanced Biliary Tract Cancer: A Network Meta-analysis. J Cancer10: 257-266.

17. Larsen FO, A Markussen, LV Diness and D Nielsen (2018) Efficacy and Safety of Capecitabine, Irinotecan, Gemcitabine, and Bevacizumab as Second-Line Treatment in Advanced Biliary Tract Cancer: A Phase II Study. Oncology 94: 19-24.

18. Arkenau HT, J Martin-Liberal, E Calvo, N Penel, MG Krebs, et al. (2018) Ramucirumab Plus Pembrolizumab in Patients with Previously Treated Advanced or Metastatic Biliary Tract Cancer: Nonrandomized, Open-Label, Phase I Trial (JVDF). Oncologist 23:1407-e136.

19. Lan CY, Y Wang, Y Xiong, JD Li, JX Shen, YF Li, et al. (2018) Apatinib combined with oral etoposide in patients with platinumresistant or platinum-refractory ovarian cancer (AEROC): phase 2, single-arm, prospective study. Lancet Oncol 19: 1239-1246.

20. Li YH, Y Zhou, YW Wang, L Tong, RX Jiang, L Xiao, et al. (2018) Comparison of apatinib and capecitabine (Xeloda) with capecitabine (Xeloda) in advanced triple- negative breast cancer as third-line therapy: A retrospective study. Medicine 355(Baltimore) 97: e12222.

21. Hu X, J Cao, W Hu, C Wu, Y Pan, L Cai, et al. (2014) Multicenter phase II study of apatinib in non-triple-negative metastatic breast cancer. BMC Cancer 14: 820.

22. Zhou N, C Liu, H Hou, C Zhang, D Liu, G Wang, K Liu, J Zhu, et al. (2016) Response to apatinib in chemotherapy-failed advanced spindle cell breast carcinoma. Oncotarget 7: 7237372379 .

23. Li F, T Zhu, B Cao, J Wang, and L Liang (2017) Apatinib 
enhances antitumour the activity of EGFR-TKIs in non-small cell lung cancer with EGFR-TKI resistance. Eur J Cancer 84: 184-192.

24. Xu J, X Liu, SYang, X Zhang, and Y Shi (2018) Clinical response to apatinib monotherapy in advanced non-small cell lung cancer. Asia Pac J Clin Oncol 14: 264-269.

25. Kong Y, L Sun, Z Hou, Y Zhang, P Chen, Y Cui, X Zhu, T Song (2017) Apatinib is effective for treatment of advanced hepatocellular carcinoma. Oncotarget 8: 105596-105605.

26. Liu C, W Xing, T Si, H Yu, and Z Guo (2017) Efficacy and safety of apatinib combined with transarterial chemoembolization for hepatocellular carcinoma with portal venous tumor thrombus: a retrospective study. Oncotarget 8: 100734-100745.

27. Yang C. and S Qin (2018) Apatinib targets both tumor and endothelial cells in hepatocellular carcinoma. Cancer Med, 7: 4570-4583.

28. Zhu B, J Li, Q Xie, L Diao, L Gai, and W Yang (2018) Efficacy and safety of apatinib monotherapy in advanced bone and soft tissue sarcoma: An observational study. Cancer Biol Ther 19: 198-204.

29. Liao Z, F Li, C Zhang, L Zhu, Y Shi, G Zhao, et al. (2019) Phase II trial of VEGFR2 inhibitor apatinib for metastatic sarcoma: focus on efficacy and safety. Exp Mol Med, 51: 24.

30. Li F, Z Liao, C Zhang, J Zhao, R Xing, S Teng, J Zhang, et al. (2018) Apatinib as targeted therapy for sarcoma. Oncotarget 9: 24548-24560.

31. Lin Y, C Wang, W Gao, R Cui, and J Liang (2017) Overwhelming rapid metabolic and structural response to apatinib in radioiodine refractory differentiated thyroid cancer. Oncotarget 8: 42252-42261.

32. Feng H, X Cheng, J Kuang, L Chen, S Yuen, M Shi, J Liang, B Shen, et al. (2018) Apatinib-induced protective autophagy and apoptosis through the AKT-mTOR pathway in anaplastic thyroid cancer. Cell Death Dis 9: 3931030.

33. Benavides M, A Anton, J Gallego, MA Gomez, A JimenezGordo, A La Casta, et al. (2015) Biliary tract cancers: SEOM clinical guidelines. Clin Transl Oncol 17: 982-987.
34. Lamarca A, RA Hubner, W David Ryder, and JW Valle (2014) Second-line chemotherapy in advanced biliary cancer: a systematic review. Ann Oncol 25: 2328-38.

35. Fornaro L, C Vivaldi, S Cereda, F Leone, G Aprile, S Lonardi, N Silvestris, et al. (2015) Second-line chemotherapy in advanced biliary cancer progressed to first-line platinum-gemcitabine combination: a multicenter surveyand pooled analysis with published data. J Exp Clin Cancer Res 34: 156.

36. Peng H, Q Zhang, J Li, N Zhang, Y Hua, L Xu, et al. (2016) Apatinib inhibits VEGF signaling and promotes apoptosis in intrahepatic cholangiocarcinoma. Oncotarget 7: 17220-17229.

37. Huang M, B Huang, G Li, and S Zeng (2018) Apatinib affect VEGF-mediated cell proliferation, migration, invasion via blocking VEGFR2/RAF/MEK/ERK and PI3K/AKT pathways in cholangiocarcinoma cell. BMC Gastroenterol, 18: 169.

38. Wang LY, S Gong, LP Gao, LX Hou, and W He (2018) Apatinib for treating advanced intrahepatic cholangiocarcinoma after failed chemotherapy: A case report and literature review. Medicine (Baltimore) 97: e13372.

39. Brieau B, L Dahan, Y De Rycke, T Boussaha, P Vasseur, et al. (2015) Second-line chemotherapy for advanced biliary tract cancer after failure of the gemcitabine-platinum combination: A large multicenter study by the Association des GastroEnterologues Oncologues. Cancer 121: 3290-3297.

40. Ahn DH and T Bekaii-Saab (2017) Biliary cancer: intrahepatic cholangiocarcinoma vs. extrahepatic cholangiocarcinoma vs. gallbladder cancers: classification and therapeutic implications. J Gastrointest Oncol 8:293-301.

41. Cardinale V, G Carpino, L Reid, E Gaudio, and D Alvaro (2012) Multiple cells of origin in cholangiocarcinoma underlie biological, epidemiological and clinical heterogeneity. World J Gastrointest Oncol 4: 94-102.

42. Chen HX and JN Cleck (2009) Adverse effects of anticancer agents that target the VEGF pathway. Nat Rev Clin Oncol 6: 465477.

43. Beauchemin C, JB Johnston, M Lapierre F Aissa and J Lachaine (2015) Relationship between progression-free survival and overall survival in chronic lymphocytic leukemia: a literature-based analysis. Curr Oncol 22: e148-56. 
44. Forsythe A, D Chandiwana, J Barth, M Thabane, J Baeck, A Shor, and G. Tremblay (2018) Is progression-free survival a more relevant endpoint than overall survival in first-line HR+/HER2metastatic breast cancer? Cancer Manag Res 10:1015-1025.

Submit your manuscript to a JScholar journal and benefit from:

ब Convenient online submission

ฯ Rigorous peer review

- Immediate publication on acceptance

- Open access: articles freely available online

ฯ High visibility within the field

- Better discount for your subsequent articles

Submit your manuscript at http://www.jscholaronline.org/submit-manuscript.php 